\title{
Heat flow dynamics in thermal systems described by diffusive representation
}

\author{
Manuel Dominguez-Pumar, Senior Member, IEEE, Maria-Teresa Atienza, Lukasz Kowalski, Santiago Novio, Sergi \\ Gorreta, Student Member, IEEE, Vicente Jimenez, Santiago Silvestre, Senior Member, IEEE
}

\begin{abstract}
The objective of this paper is to analyze the dynamics of heat flow in thermal structures working under constant temperature operation. This analysis is made using the tools of sliding mode controllers. The theory is developed considering that the thermal system can be described using diffusive representation. The experimental corroboration has been made with a prototype of a wind sensor for Mars atmosphere being controlled by a thermal sigma-delta modulator. This sensor structure allows to analyze experimentally the timevarying case since changes in wind conditions imply changes in the corresponding thermal models. The diffusive symbols of the experimental structures have been obtained from openloop measurements in which pseudo-random binary sequences of heat are injected in the sensor. With the proposed approach it is possible to predict heat flux transient waveforms in many applications.
\end{abstract}

Index Terms-diffusive representation, heat flow, thermal anemometry, sliding control, fractional order operators

\section{INTRODUCTION}

The analysis of the dynamics of heat flow in thermal structures is important in many areas such as Fuel-Cell based systems, [1], motors, [2], [3], Scanning Thermal Microscopy or Differential Scanning Calorimetry, [4]. In Scanning Thermal Microscopy, for example, nanotopography imaging is achieved by tracking temperature or heat flow changes in large arrays of cantilevers with integrated heater-thermometers kept at constant temperature, [5]. The heating can also operate in open-loop, [6], so that a constant power is injected into the heaters while the temperature of the cantilevers is monitored. Changes in the thermal conductance between the cantilever and the substrate beneath the cantilever tip can therefore be monitored by following the temperature of the cantilever. Constant temperature operation is generally preferred, in order to avoid interference with the environment of the sample, [7]. In this mode, the heat flow is the signal providing the topographical information of the sample.

Another area of interest is thermal anemometry, [8]-[12]. The main objective in thermal anemometers is to measure the convection heat transfer to a fluid in order to infer its velocity. In this work we will use, as a test structure, a first prototype made with two hemispheres of a spherical wind anemometer for Mars atmosphere, described elsewhere [9].

Both open and closed-loop strategies can be implemented on these sensors. In the open loop configuration a constant

Micro and Nano Technologies Group, Universitat Politècnica de Catalunya, Barcelona, Spain

Corresponding author: Manuel Dominguez-Pumar (email: manuel.dominguez@upc.edu heat is injected in a part of the sensor structure while the resulting temperature is being monitored. In the closed loop configuration a control circuit is used to keep this temperature constant by adapting the heat injection.

The objective of this paper is to analyze the dynamics of heat flow under constant temperature operation (closed loop configuration) in thermal structures described by models of arbitrary order. This will be done using two different analytical tools: Diffusive representation (DR) and the theory of sliding mode controllers (SMC). Diffusive representation is a theory that allows to approximate rational and non-rational convolution operators, [13]-[15]. In particular, it has proven to be very well suited for practical thermal model extraction. This is an important point since the final sensor response depends on the complete description of the thermal filter. In this regard, DR is an excellent tool that allows to approximate even fractional order systems, in practical implementations.

On the other hand, SMCs are ubiquitous to many applications [16]. These nonlinear controllers alter the dynamics of the system by applying a discontinuous control signal so that under some conditions the system 'slides' on a certain control surface. This is generally done in order to obtain a certain wanted behaviour of the system.

The study of the dynamics will be undertaken considering that the thermal controller is a sigma-delta modulator. Sigma-delta modulators have been used as analog-to-digital converters for several decades [17]. They are a class of converters producing a sequence of symbols generated from an analog signal, constituting an analog-to-digital conversion. The discrete-time iterative maps that sigma-delta modulators produce can explain the noise-shaping characteristics of these modulators. However, the dynamics of heat flow is better analyzed in what is usually called the infinite sampling frequency approximation (sampling frequency higher that the effective bandwidth of the thermal filter). It will be shown in this paper that heat flow in sensors can be understood as a Filippov solution of a sliding mode controller.

The main novelty of this paper resides in the fact that the analysis is valid for thermal filters described by any arbitrary number of poles, hence extending the findings in [8]-[12].

This paper will first provide a summary of closed-loop anemometry in section II. Section III will present a brief review of the theory of diffusive representation. Section IV will present the results on the inference of thermal models from the sensor test structure. The analysis of the dynamics with these models is divided in two parts. Section V presents the analysis of the dynamics of heat flow using the tools of SMCs. 


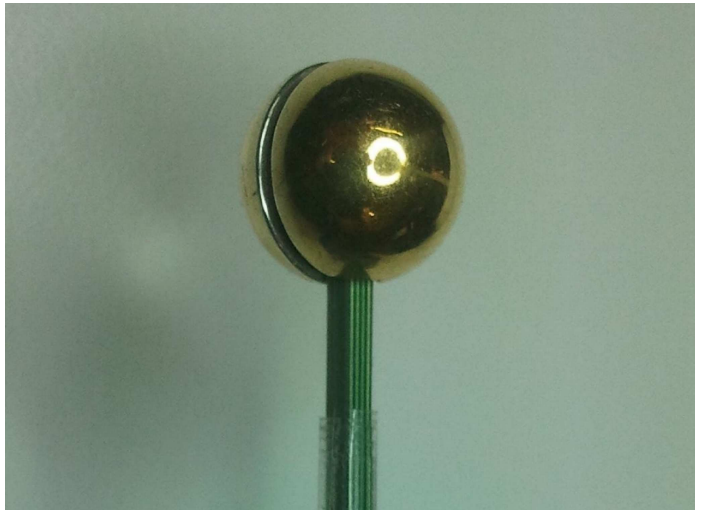

Figure 1. Photograph of the prototype of the spherical sensor anemometer.

Section VI shows that the iterative maps associated with thermal sigma-delta modulators, working with arbitrary order thermal filters, obey the general equations of standard sigmadelta modulators. Finally the experimental corroboration is presented in Section VII.

\section{CLOSED-LOOP OPERATION OF THERMAL ANEMOMETERS}

As it has been previously mentioned, thermal sensors/actuators may work in an open or a closed-loop configuration mode. In the first case, also called Constant Power Anemometry (CPA), [18], a constant power is injected in a part of the sensor structure. The output of the sensor is the resulting temperature at that point. Changes in the convection heat transfer to the fluid generate changes in temperature, which is the output signal. Using this approach, the dynamic response of the sensor is directly determined by the thermal circuits associated with the physical structure of the sensor. These thermal circuits can be very slow.

Another control variant was proposed for thermal anemometers, [18], [19], in which the temperature of a part of the sensor structure (or a temperature difference) is kept constant and the output signal is the necessary heat flow to keep this temperature constant. The main purpose of this approach is to increase the sensor bandwidth, [20], [21]. Intuitively, and as a zero-th order approximation, a constant temperature operation of a single pole thermal circuit keeps constant the value in all its nodes, [8]. Therefore, changes in the convection heat transfer must be immediately compensated by changing the heat flow into the structure. This implies that the sensor bandwidth is limited only by the actuation electronics and not by the thermal structure. The general case, though, may include thermal structures which must be described by more than one pole. For example, in [20], [21] the dynamics of hotwire anemometers working in CTA mode is analyzed up to a third order.

The experimental part of this work has been carried out using a prototype of a spherical wind sensor, [9]. Although the sensor was designed to operate in Mars atmosphere, it may also operate in Earth applications such as in the stratosphere or in wind power generation. As it can be seen in Figure 1, this

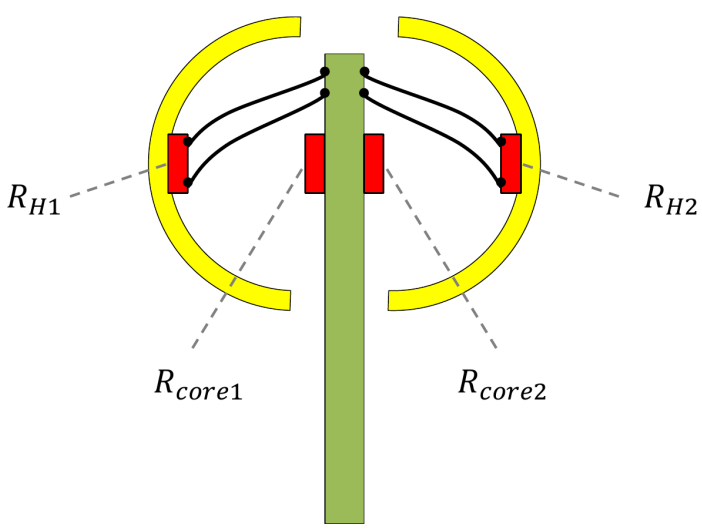

Figure 2. Schematics of the sensor anemometer showing the placement of the Pt resistors within the structure.

spherical sensor is conformed by two hemispheres connected to a PCB, providing mechanical support and the electrical connections between the sensor and the read-out circuit. These hemispheres were fabricated in silver using a 3D stereolithography printing process. A polishing is made in order to reduce emisivity [22] and finally a $100 \mathrm{~nm}$ gold layer is sputtered on top of their surface in order to avoid the oxidation of the silver.

Figure 2 shows the scheme of the sensor assembly. A $100 \Omega$ RTD sensor is integrated in each hemisphere $\left(R_{H 1}\right.$ and $\left.R_{H 2}\right)$ allowing to heat them and sense their temperature. In the normal operation mode of this sensor, both hot elements are maintained at a constant temperature target (above the air temperature) by a closed loop circuit while monitoring the electrical power needed to obtain such target. At the same time, two more RTD are attached to the PCB, $R_{\text {corel }}$ and $R_{\text {core2 }}$, in order to heat the core of the sphere, where the wires connecting $R_{H 1}$ and $R_{H 2}$ are soldered. These heaters are maintained at the same temperature as the hemispheres, thus reducing the conduction heat flux between the hemispheres and the supporting structure. The aim of this approach is to make conduction losses negligible compared with those produced by convection.

\section{REVIEW OF VARIABLE-ORDER MODELING OF CONVOLUTION OPERATORS USING DIFFUSIVE REPRESENTATION}

Thermal systems belong to the class of causal convolution systems, $H(\partial t)$, understood as operators, such that given an input signal $u$ provide an output of the form:

$$
u \longmapsto H(\partial t) u:[H(\partial t) u](t)=\int_{0}^{t} h(t-s) u(s) d s
$$

and the Laplace transform of the corresponding impulse response is $H(p)=(\mathcal{L} h)(p)$.

Diffusive Representation is a theory devoted to obtain exact and approximate state realizations of a wide class of integral operators, [13], [23], that may be of rational or non-rational nature. When it exists, a unique mathematical object, called $\gamma$-symbol, is associated with the operator, where $\gamma$ is a closed 
simple arc in $\mathbb{C}^{-}$dividing the complex plane in two sets: its exterior domain $\Omega_{\gamma}^{+}$, and, $\Omega_{\gamma}^{-}=\mathbb{C} \backslash \overline{\Omega_{\gamma}^{+}}$. In particular $H(p)$ must be holomorphic in $\Omega_{\gamma}^{+}$. Under some additional conditions it can be shown that the response of the system to an excitation $u$ can be described as:

$$
\begin{array}{ll}
\partial_{t} \psi(t, \xi) & =\gamma(\xi) \psi(t, \xi)+u(t), \psi(0, .)=0 \\
(H(\partial t) u)(t) & =\langle\eta, \psi(t, .)\rangle_{\Delta_{\gamma}^{\prime}, \Delta_{\gamma}}
\end{array}
$$

where $\xi \in \mathbb{R}$ and $\eta \in \Delta_{\gamma}^{\prime}$ is the diffusive $\gamma$-symbol, with $\Delta_{\gamma}^{\prime}$ being the dual of the vector space, $\Delta_{\gamma}$, to which all functions $\psi(\xi, t) \in \Delta_{\gamma}$ belong, [13, p.59]. In the case of thermal systems all the poles of $H(p)=H\left(\partial_{t}\right)$ are placed on the negative region of the real axis. Taking this into account we may use the following arc:

$$
\gamma(\xi)=|\xi| e^{j \operatorname{sgn}(\xi)\left(\pi-0^{+}\right)}, \quad \xi \in \mathbb{R}
$$

which clearly allows to place any poles in the negative real axis within the region $\Omega_{\gamma}^{-} \subset \mathbb{C}$, [23]. Taking this path into account, any thermal system (1) may be expressed in a state space realization of the form:

$$
\begin{array}{ll}
\partial_{t} \psi(t, \xi) & =-\xi \psi(t, \xi)+u(t) \\
(H(\partial t) u)(t) & =\int_{0}^{\infty} \eta(\xi) \psi(\xi, t) d \xi
\end{array}
$$

with $\xi \geq 0$ and $\psi(0,)=$.0 . Let us assume that $\mathcal{M}$ is a Hilbert subspace of $\Delta_{\gamma}^{\prime}$, and that $\mathcal{Q}$ is a Hilbert subspace of $\Delta_{\gamma}$ containing $H(p)$. We may define an injective operator $\mathfrak{U}_{\gamma}: \mathcal{M} \rightarrow \mathcal{Q}$, such that if $\eta \in \mathcal{M}$ then:

$$
\mathfrak{U}_{\gamma}(\eta)=\int_{0}^{\infty} \frac{\eta(\xi)}{p+\xi} d \xi
$$

and the associated frequency response is:

$$
H(\Omega)=\int_{0}^{\infty} \frac{\eta_{H}(\xi)}{j \Omega+\xi} d \xi
$$

where we have that $\mathfrak{U}_{\gamma}\left(\eta_{H}\right)=H(p)$. It must be noted that since $\xi$ is a continuous variable it is possible to handle infinite order systems, such as in the case of fractional operators.

\section{A. Numerical approximations}

Under some conditions it is possible to construct finite order approximations of fractional order operators arbitrarily close to the original operator $H(\partial t) u$, [13].

1) Finite order approximation of operators from the discretization of $\gamma$-symbols: Given a sequence of L-dimensional subspaces of atomic measures, $\mathcal{M}_{L} \subset \mathcal{M}$, defined on certain meshes, $\left\{\xi_{l}\right\}, l=1 . . L$, of the $\xi$ variable, if $\bigcup_{L} \mathcal{M}_{L}$ is dense in $\mathcal{M}$ (or what is the same the union of the supports of the meshes is dense in $\left.\mathbb{R}: \overline{\cup_{L} \operatorname{Supp}\left(\mathcal{M}_{L}\right)}=\mathbb{R}\right)$ and if:

$$
\eta_{L} \underset{L \rightarrow \infty}{\longrightarrow} \eta
$$

in the strong sense, then we have that $\mathfrak{U}_{\gamma}\left(\eta_{L}\right) \rightarrow \mathfrak{U}_{\gamma}(\eta)$, [13, p. 83]. This means that good finite-order approximations of $\gamma-$ symbols provide good approximations of the corresponding operator.
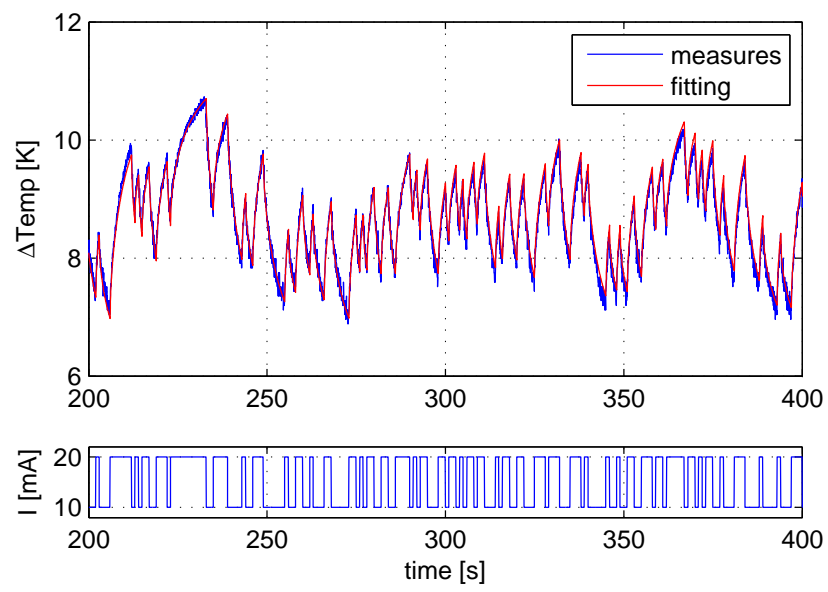

Figure 3. Experiment carried out to infer the diffusive symbol associated with the structure. Bottom: current injected into the Pt heater $\left(I_{\text {low }}=10 \mathrm{~mA}\right.$ and $I_{\text {high }}=20 \mathrm{~mA}$ ). Top: time evolution of the temperature of the Pt resistor, in blue, with the corresponding 7 poles model obtained with the optimization procedure based on diffusive representation, in red.

In the case of thermal operators, the finite-order approximations of $\gamma$-symbols are of the form:

$$
\begin{array}{ll}
\partial_{t} \psi_{k}(t) & =-\xi_{k} \psi_{k}(t)+u(t) \\
\left(H_{L}(\partial t) u\right)(t) & =\sum_{k=1}^{L} \eta_{k} \psi_{k}(t)
\end{array}
$$

for $k=1, \cdots, L$ and with $\psi_{k}(0)=0$. How good the approximation can be will depend on how the frequency mesh $\xi_{k}$ has been chosen. In thermal systems it is usual to make a geometric frequency mesh, within the band of interest, [24].

\section{B. Inference of finite order approximations of $\gamma$-symbols of operators}

Under some conditions finite-order approximations can be obtained from pseudo-inverting the $\mathfrak{U}_{\gamma}$ operator $\left(\mathfrak{U}_{\gamma}{ }^{*} \mathfrak{U}_{\gamma}\right)^{-1} \mathfrak{U}_{\gamma}{ }^{*}:$

$$
\eta_{\mathrm{opt}}^{n}=\underset{\eta_{n} \in \mathcal{M}_{n}}{\arg \min }\left\|\int_{0}^{\infty} \frac{\eta_{n}(\xi)}{p+\xi} d \xi-H(p)\right\|_{\mathcal{Q}}
$$

If the operator $\mathfrak{U}_{\gamma}{ }^{-1}$ is bounded, then the solution of the above problem is strongly convergent to $\eta$. However, this is not generally the case, [13]. Nevertheless, stable optimal approximations can be obtained using a convenient penalization: $\left(\mathfrak{U}_{\gamma}{ }^{*} \mathfrak{U}_{\gamma}+\epsilon I\right)^{-1} \mathfrak{U}_{\gamma}{ }^{*}$.

\section{Practical inference of DyNamical models BASED ON DIFFUSIVE REPRESENTATION}

A number of works have been focused on obtaining diffusive symbols of operators by means of measurements. The measurements have been made in time, [24], or in the frequency domains, [25], [26]. In this work we have opted for time-domain characterizations of the thermal system.

A predetermined current sequence is injected at the $\mathrm{Pt}$ resistor of one of the hemispheres (open loop actuation). This sequence is based on a Pseudo Random Binary Sequence 


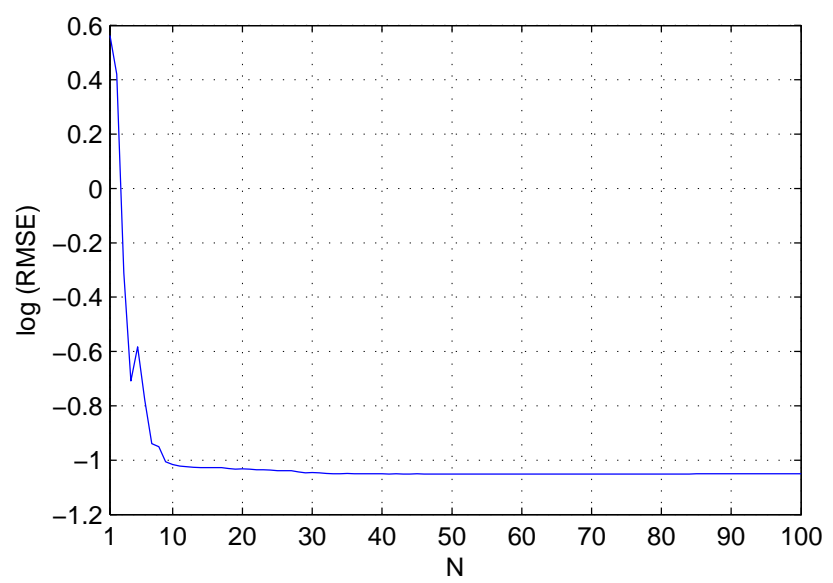

Figure 4. Evolution of the Root Mean Square Error of the pseudo-inversions corresponding the measurements obtained with the sensor structure, as a function of the order of the model.

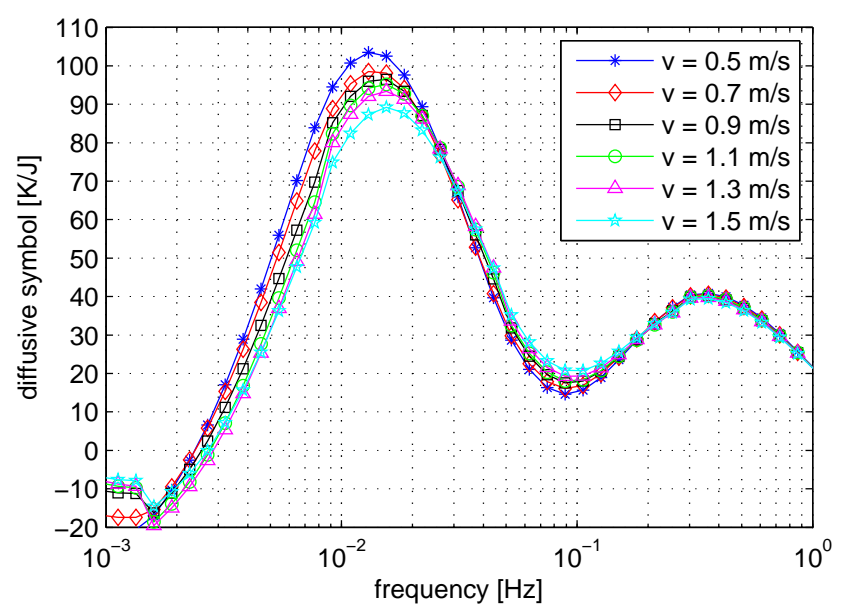

Figure 5. Zoom of the 70-pole diffusive symbols resulting from 10 geometric shifts of 7-order models obtained from pseudoinversion of experimental measurements of the sensor structure. Each curve corresponds to a different wind velocity

(PRBS). The core and the other hemisphere are controlled to keep a constant target temperature. This condition is interesting in the case of anemometers where it is important to reduce the heat conduction to the structure, at least compared to heat convection. If the structure is always kept at the same temperature, as a first order approximation, conduction losses can be neglected.

We focus now on the results obtained for six wind speeds: $0.5,0.7,0.9,1.1,1.3$ and $1.5 \mathrm{~m} / \mathrm{s}$. For each wind speed an open loop experiment has been carried out with a duration of 1 hour. The temperature of the corresponding $\mathrm{Pt}$ resistor is sampled at $f_{S}=20 \mathrm{~Hz}$. The 'bit' period of the 12-bit PRBS sequence is $1 \mathrm{~s}$.

As an example, Figure 3 shows the waveform obtained with an experiment made under low wind velocity condition: 0.5 $\mathrm{m} / \mathrm{s}$. Figure 4 shows the evolution of the root mean square error of the fittings as a function of the model order. No appreciable gain is obtained beyond $\mathrm{N}=10$.
Figure 5 shows a zoom in the range $f \in\left[10^{-3}, 1\right][\mathrm{Hz}]$ of the diffusive symbols of the sensor for different wind velocity conditions. These models have been obtained from 10 frequency mesh shifts of models of order 7 .

With this approach then it is possible to infer diffusive symbols with higher number of poles. On the other hand we have observed that diffusive symbols of 7 poles are sufficient to obtain a good match with the open-loop response of the sensor and allows to distinguish among different wind velocity conditions.

\section{CONSTANT TEMPERATURE OPERATION: THERMAL SIGMA-DELTA MODULATION}

We are interested in the analysis of the heat flow in thermal structures under constant temperature operation. In order to enforce a constant operation many different possible controls can be implemented. In this work we will focus on firstorder thermal sigma-delta modulation since it presents various advantages for the implementation of Constant Temperature Anemometry, [8]. This modulation is a very simple discretetime control in which at each sampling period, $T_{S}$, the current temperature of the structure, $T_{n}=T\left(n T_{S}\right)$, is compared with the desired target temperature, $\Delta T$.

- If $T_{n} \geq \Delta T$ then the heater is switched OFF during the following sampling period ( $P_{\text {off }}$ is injected into the heater),

- If $T_{n}<\Delta T$ then the heater is switched on during the following sampling period $\left(P_{\text {on }}\right.$ is injected into the heater).

In order to be able to adapt the sensor to different flow and temperature conditions it is usual to have a quiescent power: $P_{\text {off }} \neq 0$.

Now, let us analyze the dynamics of the system for general case of finite order thermal systems. Under the infinite sampling approximation and for a constant wind, the bitstream $b_{n}$ may be seen as a continuous time variable:

$$
b_{n} \underset{T_{S} \rightarrow 0}{\longrightarrow} b(t)=\frac{\operatorname{sgn}(\sigma(t))}{2}\left(P_{\text {on }}-P_{\text {off }}\right)+\frac{1}{2}\left(P_{\text {on }}+P_{\text {off }}\right)
$$

where $\sigma(t)=\Delta T-\left[H\left(\partial_{t}\right) b\right](t)$. Taking into account the finite set of frequencies of the thermal filter, $\left\{\xi_{k}\right\}$, the discrete time map now becomes:

$$
\partial_{t} \psi_{k}(t)= \begin{cases}-\xi_{k} \psi_{k}(t)+P_{\text {on }}, & \sigma>0 \\ -\xi_{k} \psi_{k}(t)+P_{\text {off }}, & \sigma<0\end{cases}
$$

If we define $\Psi(t)=\left(\psi_{1}(t), \cdots, \psi_{N}(t)\right)^{T} \in \Delta_{\gamma} \equiv \mathbb{R}^{N}$ and using the diffusive symbol we have:

$$
\begin{aligned}
\sigma(\Psi(t))=\Delta T & -\langle\eta, \Psi(t)\rangle_{\Delta_{\gamma}^{\prime}, \Delta_{\gamma}}= \\
& =\Delta T-\sum_{k}^{N} \eta_{k} \psi_{k}(t)
\end{aligned}
$$




\section{A. Reachability conditions}

The obvious objective of a constant temperature operation is to place the thermal system within the control surface $\sigma(\Psi(t))=0$, i.e., $\left[H\left(\partial_{t}\right) b\right](t)=\Delta T$, or what is the same to have $\Psi(t) \in \mathcal{S}$ where $\mathcal{S}:=\left\{f \in \Delta_{\gamma}: \sigma(f)=0\right\}$.

However, depending on the maximum and minimum available heat injections, $P_{\text {on }}$ and $P_{\text {off }}$, this may or may not be possible. First, we see that, regardless of the initial condition of the system, in finite time or asymptotically:

$$
\psi_{k}(t) \in\left[\frac{P_{\text {off }}}{\xi_{k}}, \frac{P_{\text {on }}}{\xi_{k}}\right]
$$

The thermal system will reach the control surface $\mathcal{S}$ in finite time if:

$$
P_{\text {off }} \sum_{k}^{N} \frac{\eta_{k}}{\xi_{k}}<\Delta T<P_{\text {on }} \sum_{k}^{N} \frac{\eta_{k}}{\xi_{k}}
$$

This follows from the fact that the control surface $\sigma=$ 0 divides in two parts the state space. On one side of the hyperplane, $\sigma>0$, the system evolution is:

$$
\partial_{t} \psi_{k}(t)=-\xi_{k} \psi_{k}(t)+P_{\text {on }}
$$

The above equation, for each $\xi_{k}$, simply describes a linear system actuated with a constant control. Therefore we have that:

$$
\psi_{k}(t)=\psi_{k}(0) e^{-\xi_{k} t}+\frac{P_{\mathrm{on}}}{\xi_{k}}\left(1-e^{-\xi_{k} t}\right), \quad \text { for } t>0
$$

This means that from any initial $\Psi(0)$ such that $\sigma(\Psi(0))>0$ the control surface $\sigma=0$ will be reached if the asymptotic point of this trajectory, $\psi_{k}(\infty)=\frac{P_{\text {on }}}{\xi_{k}}$, lies on the other side of the hyperplane, i.e., we have the second inequality of condition (14). A similar analysis can be carried out for the case where the initial condition lies in the region $\sigma<0$, obtaining the first inequality of expression (14).

This means that if conditions (14) are fulfilled, the control surface $\mathcal{S}$, will be continuously reached in time (there is no $t_{0}$ such that for all $t>t_{0}$ it is $\left.\psi_{k}(t) \notin \mathcal{S}\right)$. This amounts to not having an asymptotically stable equilibrium point of the system $\dot{\psi}_{k}(t)=-\xi_{k} \psi_{k}(t)+P_{\text {on }}$, (resp. $\dot{\psi}_{k}(t)=-\xi_{k} \psi_{k}(t)+$ $P_{\text {off }}$ ), inside the set $\sigma>0$, (resp. $\left.\sigma<0\right)$.

\section{B. Attractive sliding region within the control surface $\mathcal{S}$}

In this section we will apply the techniques used for obtaining fast switching in relay feedback systems that can be found in [27] and [28]. This will provide us with conditions that guarantee the existence of a sliding region within the control surface. First, we must note that:

$$
=\left\{\begin{array}{lr}
\sum_{k}^{N} \eta_{k} \xi_{k} \psi_{k}(t)-P_{\mathrm{on}} \Gamma, & \sigma(\Psi(t))>0 \\
\sum_{k}^{N} \eta_{k} \xi_{k} \psi_{k}(t)-P_{\mathrm{off}} \Gamma, & \sigma(\Psi(t))<0
\end{array}\right.
$$

where $\Gamma=\sum_{k}^{N} \eta_{k}$. This means that the intersection of the following subset of the state space $\Delta_{\gamma}$ :

$$
\Omega:=\left\{f \in \Delta_{\gamma}: P_{\text {off }}<\frac{1}{\Gamma} \sum_{k}^{N} \eta_{k} \xi_{k} f_{k}<P_{\text {on }}\right\}
$$

with the control surface $\mathcal{S}$, i.e. $\Omega \cap \mathcal{S}$, is attractive. This is due to the fact that if $\Psi(t) \in \Omega$ and $\sigma(t)<0$ we will have $\dot{\sigma}(t)>0$, whereas if $\sigma(t)>0$ then we will have $\dot{\sigma}(t)<$ 0 . Therefore it is $\sigma \dot{\sigma} \leq 0$ in $\Omega$. This means that $\Omega \cap \mathcal{S}$, is attractive. It must be noted that if $\eta_{k} \geq 0$ conditions in (18) are always fulfilled since we have (13). This means that the reachability conditions imply that $\sigma(\infty)=0$. Intuitively we see that if the system we are analyzing measures an absolute temperature (not a difference of temperatures in two parts of a structure, for example) and we are able to reach the desired target temperature, then a thermal sigma-delta modulator will always reach an equilibrium point within the control surface.

\section{Sliding motion}

Under a sliding motion, and considering a Filippov solution with an equivalent control $u_{\text {eq }}$, we will have the condition $\dot{\sigma}=0$, or what is the same:

$$
\dot{\sigma}(t)=-\sum_{k}^{N} \eta_{k} \dot{\psi}_{k}(t)=\sum_{k}^{N} \eta_{k}\left(\xi_{k} \psi_{k}(t)-u_{\mathrm{eq}}(t)\right)=0
$$

From the last equation we find that the equivalent control, in the sense of Filippov, depends on the current value of $\psi_{k}(t)$ :

$$
u_{\mathrm{eq}}(t)=\Gamma^{-1} \sum_{k}^{N} \eta_{k} \xi_{k} \psi_{k}(t)
$$

This is the equivalent control that must be applied to the system in order to keep it on the control surface $\sigma=0$.

Considering now that the equivalent control is being applied, the dynamics of the system, within the control surface $\sigma=0$, is determined by the following set of equations for $\xi_{k}>0$ :

$$
\dot{\psi}_{k}(t)+\xi_{k} \psi_{k}(t)=\frac{1}{\Gamma} \sum_{l}^{N} \eta_{l} \xi_{l} \psi_{l}(t)
$$

If the system reaches an equilibrium point, in which $\dot{\psi}_{k}(t)=0$ we will have that $\psi_{k}(t)=\psi_{k}^{Q}$, and therefore there will be a constant equivalent control $u_{\mathrm{eq}}(t)=u_{Q}$ :

$$
\begin{array}{ll}
\psi_{\mathrm{Q}}\left(\xi_{k}\right) & =\frac{u_{Q}}{\xi_{k}}, \Delta T \\
u_{Q} & =\frac{\Delta T}{\sum_{k}^{N} \eta_{k} / \xi_{k}}
\end{array}
$$

\section{Effect of external disturbances and model uncertainties}

Let us now assume that the thermal system has some model uncertainties and external disturbances represented by an additional term, $\Theta(\Psi, t)=\left(\theta_{1}(\Psi, t), \cdots, \theta_{N}(\Psi, t)\right)^{T}$. Since the initial conditions are zero, $\Psi(0)=0$, we have that the thermal system can also be expressed as: 


$$
\begin{aligned}
\partial_{t} \psi_{k}(t)= & -\xi_{k} \psi_{k}(t)+\frac{\eta_{k}}{2}\left(P_{\text {off }}+P_{\text {on }}\right) \\
& +\frac{\eta_{k}}{2}\left(P_{\text {on }}-P_{\text {off }}\right) \operatorname{sgn} \sigma+\theta_{k}(\Psi, t) \\
\sigma(\Psi(t))= & \Delta T-\sum_{k}^{N} \psi_{k}(t)
\end{aligned}
$$

Assuming a sliding regime is taking place, so that $\partial_{t} \sigma=0$, the equivalent control is now:

$$
u_{\mathrm{eq}}=\frac{\sum_{k}^{N} \xi_{k} \psi_{k}(t)-\frac{\Gamma}{2}\left(P_{\mathrm{off}}+P_{\mathrm{on}}\right)-\chi(\Psi, t)}{\frac{\Gamma}{2}\left(P_{\mathrm{on}}-P_{\mathrm{off}}\right)} \in[-1,1]
$$

with $\chi(\Psi, t)=\sum_{k} \theta_{k}(\Psi, t)$. And the new dynamics within the control surface is then:

$$
\begin{aligned}
\partial_{t} \psi_{k}(t)= & -\xi_{k} \psi_{k}(t)+\frac{\eta_{k}}{2}\left(P_{\text {off }}+P_{\text {on }}\right)+\theta_{k}(\Psi, t) \\
& +\frac{\eta_{k}}{\Gamma}\left[\sum_{l} \xi_{l} \psi_{l}(t)-\frac{\Gamma}{2}\left(P_{\text {on }}+P_{\text {off }}\right)-\chi(\Psi, t)\right]
\end{aligned}
$$

As it can be seen, the role of a matched disturbance is played by a disturbance proportional to the diffusive symbol, i.e. $\theta_{k}(\Psi, t)=\alpha \eta_{k}$ for some $\alpha \in \mathbb{R}$, in which case $\theta_{k}(t)-\eta_{k} \chi(\Psi, t) / \Gamma=0$. Under this condition, the dynamics of the system would remain undisturbed while the equivalent control would instantaneously absorb the changes due to the disturbance.

In a more general case, any disturbance will generate a change in the dynamics of the system and also a change in the equivalent control.

\section{ITERATIVE MAPS WITH ARBITRARY ORDER THERMAL MODELS}

Once we have undertaken the sliding mode study of the system including a full thermal model, we may analyze whether for a constant wind condition, and once in the final equilibrium point, i.e. expressions (22), the dynamics associated with the discrete-time map is the one associated with a sigma-delta modulator.

$$
\dot{\sigma}= \begin{cases}\sum_{k}^{N} \eta_{k} \xi_{k} \psi_{k}(t)-\Gamma P_{\mathrm{on}}, & \sigma>0 \\ \sum_{k}^{N} \eta_{k} \xi_{k} \psi_{k}(t)-\Gamma P_{\mathrm{off}}, & \sigma<0\end{cases}
$$

which means that:

$$
\sigma_{n+1} \approx \begin{cases}\sigma_{n}+t_{S}\left(\Gamma u_{Q}-\Gamma P_{\text {on }}\right), & \sigma>0 \\ \sigma_{n}+t_{S}\left(\Gamma u_{Q}-\Gamma P_{\text {off }}\right), & \sigma<0\end{cases}
$$

and therefore:

$$
\begin{array}{r}
\sigma_{n+1}=\sigma_{n}+\Delta T \frac{\Gamma}{\sum_{k}^{N} \frac{\eta_{k}}{\xi_{k}}} t_{S} \\
-\frac{\Gamma t_{S}}{2}\left(P_{\text {off }}+P_{\text {on }}+\left(P_{\text {on }}-P_{\text {off }}\right) \operatorname{sgn}\left(\sigma_{n}\right)\right)
\end{array}
$$

Then the dynamics of the bitstream around the equilibrium corresponds to one of a sigma-delta modulator. By defining:

$$
\begin{aligned}
& u_{n}:=\frac{2}{\Gamma t_{S}} \frac{\sigma_{n}}{P_{\text {on }}-P_{\text {off }}} \\
& \delta:=2 \frac{\Delta T}{\sum_{k}^{N} \frac{\eta_{k}}{\xi_{k}}} \frac{1}{P_{\text {on }}-P_{\text {off }}}-\frac{P_{\text {off }}+P_{\text {on }}}{P_{\text {on }}-P_{\text {off }}} \in[-1,1]
\end{aligned}
$$

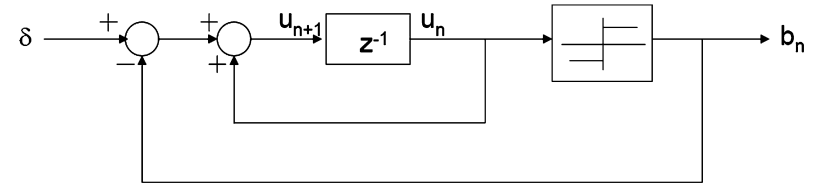

Figure 6. First-order sigma-delta modulator.

we obtain the same equation of a first-order sigma-delta modulator:

$$
u_{n+1}=u_{n}+\delta-\operatorname{sgn}\left(u_{n}\right)
$$

where $u_{n}$ is the sequence associated with the integrator of the sigma-delta modulator, see Figure 6.

\section{EXPERIMENTAL RESULTS}

The purpose of this section is to compare the experimental results obtained using the sensor structure described in Section II, with the predictions of the sliding mode analysis. Since this structure is a prototype of a wind sensor, the measurement consists in switching on the thermal sigma-delta converter to a specific target temperature, $\Delta T$, under constant wind conditions. In a first phase, the temperature is below the desired value, and the heater is constantly on. Once the control surface is reached, the second phase begins and the heater signal begins to switch in time and the typical bitstream is obtained. In the performed experiments, once the bitstream of the second phase has been stabilized, the wind velocity is changed. With this change, the thermal system and its dynamics change accordingly.

Two experiments have been carried out under different target temperatures, $\Delta T$, and different wind velocity conditions. In both experiments, the temperature controllers of one of the hemispheres and the core keep at the same constant temperature their respective Pt heaters. At $t=0$ the remaining controller is switched on.

In experiment I, the wind is changed from 0.8 to $0.3 \mathrm{~m} / \mathrm{s}$. The target temperature is $\Delta T=4.7 \mathrm{~K}$. On the other hand, experiment II has been carried out using six different wind velocities. The wind is changed in steps of $0.2 \mathrm{~m} / \mathrm{s}$ from 0.5 to $1.5 \mathrm{~m} / \mathrm{s}$ and when the maximum wind velocity is reached, the wind velocity is switched back to $0.5 \mathrm{~m} / \mathrm{s}$ again. The target temperature in this case is $\Delta T=6.9 \mathrm{~K}$.

The wind velocities used in both experiments then, have ranged from 0.3 to $1.5 \mathrm{~m} / \mathrm{s}$. The experiments were made at room temperature and the air pressure was set to $45 \mathrm{mbar}$. Taking into account the particular geometry of the sensor, the obtained Reynolds numbers range from 8.4 to 42.2. Under typical Martian conditions (6 mbar, $\mathrm{CO}_{2}$ and $220 \mathrm{~K}$ ) [29], the range of velocities that provide the same Reynolds numbers go from 0.65 to $3.25 \mathrm{~m} / \mathrm{s}$.

The experimental control waveforms can be observed in Figures 7 and 8 and there is good agreement with the prediction made by the sliding analysis. The thermal models have been inferred from PRBS measurements as explained in Section IV for eight wind velocities: $0.3,0.5,0.7,0.8,0.9,1.1$, 1.3 and $1.5 \mathrm{~m} / \mathrm{s}$. In the sliding mode analysis, first, the point at which the system reaches the control surface is calculated. 


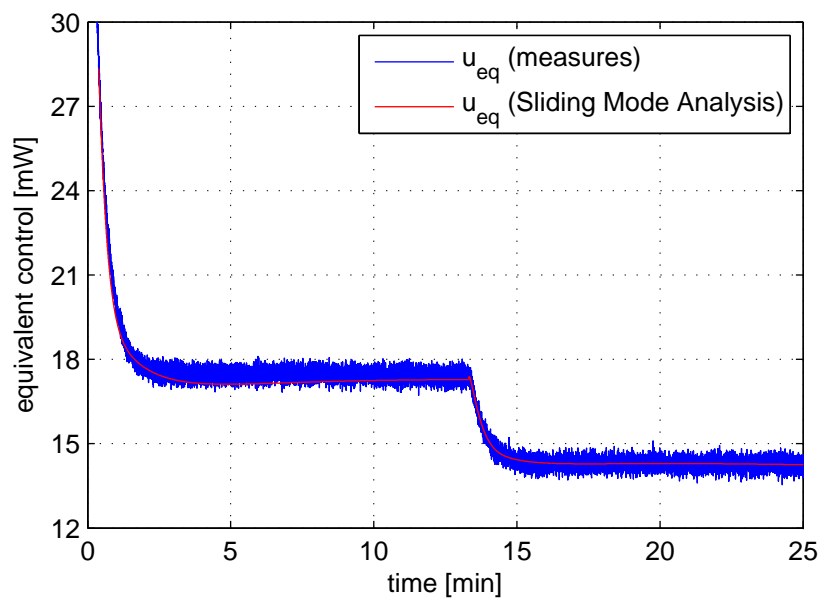

Figure 7. Equivalent control of experiment I. In this case the temperature of the core and one of the hemispheres is kept at $\Delta T=4.7 \mathrm{~K}$. At $t=0$ the controller of the remaining hemisphere is switched on $(\Delta T=4.7 \mathrm{~K}$, $\Sigma-\Delta$ clock frequency $20 \mathrm{KHz}$, average power samples obtained from 1000 consecutive sampling periods). At $\mathrm{t}=800 \mathrm{~s}$ the wind is changed from $0.8 \mathrm{~m} / \mathrm{s}$ to $0.3 \mathrm{~m} / \mathrm{s}$. The blue curve shows the experimental average power injected into the heater. The red curve shows the result of applying the sliding mode analysis to this measurement using a diffusive symbol of 10 poles.

From a zero initial condition, a constant $u(t)=P_{\text {on }}$ is applied till the control surface $\sigma=0$ is reached. The intersection point of this initial trajectory with the surface, $\Psi(0) \in \mathcal{S}$, is the initial condition for the sliding movement on the control surface following expressions (20) and (21) for the first wind step of each experiment.

Once the bitstream at the output of the sigma-delta modulator is stable, the wind is changed. In experiment I, the change is produced only once at $t_{C_{1}}=800 \mathrm{~s}$, from 0.8 to $0.3 \mathrm{~m} / \mathrm{s}$. In experiment II, however, ten wind velocity changes have been made. The instants when the wind change are $t_{C_{i}}=300 i$, $i=1, \cdots, 10$.

In both experiments, the thermal sigma-delta of the hemisphere is switched on at $t_{C_{0}}=0$. As mentioned before, the sliding analysis is made taking into account equations (20) and (21). Due to the fact that the thermal filters change when the wind velocity is changed, the initial conditions for the dynamics of the system at each wind interval have to be found. These values are obtained from a fitting of the experimental measurements, with the additional affine constraint $\left(\eta^{i}\right)^{T} \Psi\left(t_{C_{i}}{ }^{+}\right)=\Delta T$, where $\left(\eta^{i}\right)^{T}$ are the diffusive symbols corresponding to the wind of the $i$-th interval.

Figures 7 and 8 show the comparison between the experimental equivalent controls generated from the two experiments, compared with the sliding mode analysis. In both cases it is possible to explain the dynamics of the sensor as a result of how it is operated and the how the wind changes during the experiments.

\section{CONCLUSIONS}

The dynamics of the heat flow of a sensor structure working at constant temperature has been analyzed using the tools of sliding mode controllers. The dynamical finite-order thermal models based on diffusive representation have been extracted

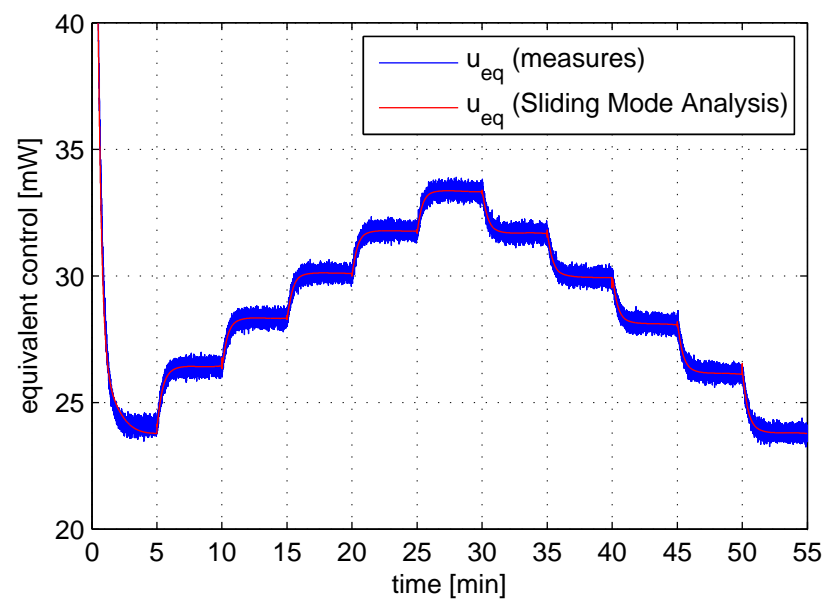

Figure 8. Equivalent control of experiment II. In this case the temperature of the core and one of the hemispheres is kept at $\Delta T=6.9 \mathrm{~K}$. At $t=0$ the controller of the remaining hemisphere is switched on $(\Delta T=6.9 \mathrm{~K}$, $\Sigma-\Delta$ clock frequency $20 \mathrm{KHz}$, average power samples obtained from 1000 consecutive sampling periods). Every $5 \mathrm{~min}$, the wind is changed in $0.2 \mathrm{~m} / \mathrm{s}$ steps. Wind speed is changed from 0.5 to $1.5 \mathrm{~m} / \mathrm{s}$ and from 1.5 to $0.5 \mathrm{~m} / \mathrm{s}$. The blue curve shows the experimental average power injected into the heater. The red curve shows the result of applying the sliding mode analysis to this measurement using a diffusive symbol of 10 poles.

from open-loop measurements using pseudo-random binary sequences. The experimental results have been obtained with a prototype of a wind sensor. With the proposed approach it is possible to understand the time evolution of the control bitstreams as a function of changes in wind velocity.

\section{REFERENCES}

[1] J. D. Rojas, C. Kunusch, C. Ocampo-Martinez, and V. Puig, "ControlOriented Thermal Modeling Methodology for Water-Cooled PEM Fuel-Cell-Based Systems,” IEEE Trans. Ind. Electron., vol. 62, no. 8, pp. 5146-5154, aug 2015.

[2] Y. C. Chong, E. J. P. Echenique Subiabre, M. A. Mueller, J. Chick, D. A. Staton, and A. S. McDonald, "The Ventilation Effect on Stator Convective Heat Transfer of an Axial-Flux Permanent-Magnet Machine," IEEE Trans. Ind. Electron., vol. 61, no. 8, pp. 4392-4403, aug 2014.

[3] H. K. Jussila, A. V. Mityakov, S. Z. Sapozhnikov, V. Y. Mityakov, and J. Pyrhonen, "Local Heat Flux Measurement in a Permanent Magnet Motor at No Load," IEEE Trans. Ind. Electron., vol. 60, no. 11, pp. 4852-4860, nov 2013.

[4] A. Nespoli, F. Passaretti, F. Stortiero, and E. Villa, "Temperaturemodulated differential scanning calorimetry for the study of reversing and nonreversing heat flow of shape memory alloys," J. Therm. Anal. Calorim., vol. 116, no. 2, pp. 771-777, jan 2014.

[5] Y. Gianchandani, "A temperature-dithering closed-loop interface circuit for a scanning thermal microscopy system," J. Microelectromechanical Syst., vol. 14, no. 1, pp. 44-53, feb 2005.

[6] K. Kim, K. Park, J. Lee, Z. Zhang, and W. King, "Nanotopographical imaging using a heated atomic force microscope cantilever probe," Sensors Actuators A Phys., vol. 136, no. 1, pp. 95-103, may 2007.

[7] S. Somnath and W. P. King, "Heated atomic force cantilever closed loop temperature control and application to high speed nanotopography imaging," Sensors Actuators A Phys., vol. 192, pp. 27-33, apr 2013.

[8] M. Dominguez-Pumar, V. Jimenez, J. Ricart, L. Kowalski, J. Torres, S. Navarro, J. Romeral, and L. Castañer, "A hot film anemometer for the Martian atmosphere," Planet. Space Sci., vol. 56, pp. 1169-1179, 2008.

[9] L. Kowalski, M. T. Atienza, S. Gorreta, V. Jiménez, M. DomínguezPumar, S. Silvestre, and L. M. Castañer, "Spherical wind sensor for the atmosphere of mars," IEEE Sensors Journal, vol. 16, no. 7, pp. 18871897, April 2016. 
[10] K. A. A. Makinwa and J. H. Huijsing, "A wind-sensor interface using thermal sigma delta modulation techniques," Sensors Actuators A Phys., vol. 92, no. 1-3, pp. 280-285, 2001.

[11] - "A smart wind sensor using thermal sigma-delta modulation techniques," Sensors Actuators A Phys., vol. 97-98, no. 0, pp. 15-20, 2002.

[12] W. R. M. Almeida, G. M. Freitas, L. S. Palma, S. Y. C. Catunda, R. C. S. Freire, F. F. Santos, A. Oliveira, and H. Aboushady, "Thermal sigmadelta modulator: Anemometer performance analysis," in Instrumentation and Measurement Technology Conference Proceedings, 2007. IMTC 2007. IEEE, May 2007, pp. 1-5.

[13] G. Montseny, Representation diffusive. Lavoisier, 2005.

[14] K. Ben Smida, P. Bidan, T. Lebey, F. Ben Ammar, and M. Elleuch, "Identification and Time-Domain Simulation of the Association Inverter-Cable-Asynchronous Machine Using Diffusive Representation," IEEE Trans. Ind. Electron., vol. 56, no. 1, pp. 257-265, jan 2009.

[15] C. Restrepo, G. Garcia, J. Calvente, R. Giral, and L. Martinez-Salamero, "Static and Dynamic Current-Voltage Modeling of a Proton-Exchange Membrane Fuel-Cell Using an Input-Output diffusive approach." IEEE Trans. Ind. Electron., vol. PP, no. 99, pp. 1-1, 2015.

[16] A. Leon-Masich, H. Valderrama-Blavi, J. M. Bosque-Moncusi, J. MaixeAltes, and L. Martinez-Salamero, "Sliding-Mode-Control-Based Boost Converter for High-Voltage-Low-Power Applications," IEEE Trans. Ind. Electron., vol. 62, no. 1, pp. 229-237, jan 2015.

[17] V. A. T. L. D. George I Bourdopoulos Aristodemos Pnevmatikakis, Delta-Sigma Modulators: Modeling, Design and Applications. Imperial College Press, 2003.

[18] T. Lammerink, N. Tas, G. Krijnen, and M. Elwenspoek, "A new class of thermal flow sensors using $\Delta \mathrm{T}=0$ as a control signal," in Proc. IEEE Thirteen. Annu. Int. Conf. Micro Electro Mech. Syst. (Cat. No.00CH36308). IEEE, 2000, pp. 525-530.

[19] T. Qin-Yi and H. Jin-Biao, "A novel CMOS flow sensor with constant chip temperature (CCT) operation," Sensors and Actuators, vol. 12, no. 1, pp. 9-21, jul 1987.

[20] P. Freymuth, "Interpretations in the control theory of thermal anemometers," Meas. Sci. Technol., vol. 8, no. 2, pp. 174-177, feb 1997.

[21] — " "On higher order dynamics of constant-temperature hot-wire anemometers," Meas. Sci. Technol., vol. 9, no. 3, pp. 534-535, mar 1998.

[22] S. Wladimir and R. Todtenhaupt, "Effect of roughness on the emissivity of the precious metals silver, gold, palladium, platinum, rhodium, and iridium," in 15th European Conference on Thermophysical Properties, vol. 33, Würzburg, Germany, September 1999, pp. 861-869.

[23] G. Montseny, "Simple Approach to Approximation and Dynamical Realization of Pseudodifferential Time Operators Such as Fractional Ones," IEEE Trans. Circuits Syst. II Express Briefs, vol. 51, no. 11, pp. 613-618, nov 2004.

[24] B. Allard, X. Jorda, P. Bidan, A. Rumeau, H. Morel, X. Perpina, M. Vellvehi, and S. M'Rad, "Reduced-Order Thermal Behavioral Model Based on Diffusive Representation," Power Electron. IEEE Trans., vol. 24, no. 12, pp. 2833-2846, dec 2009.

[25] J. N. Davidson, D. A. Stone, M. P. Foster, and D. T. Gladwin, "Improved Bandwidth and Noise Resilience in Thermal Impedance Spectroscopy by Mixing PRBS Signals," IEEE Trans. Power Electron., vol. 29, no. 9, pp. 4817-4828, sep 2014.

[26] K. Ben Smida, P. Bidan, T. Lebey, F. Ben Ammar, and M. Elleuch, "Identification and Time-Domain Simulation of the Association Inverter-Cable-Asynchronous Machine Using Diffusive Representation," IEEE Trans. Ind. Electron., vol. 56, no. 1, pp. 257-265, jan 2009.

[27] J. Goncalves, A. Megretski, M. A. Dahleh, J. M. Gonçalves, A. Megretski, and M. A. Dahleh, "Global stability of relay feedback systems," Autom. Control. IEEE Trans., vol. 46, no. 4, pp. 550-562, apr 2001.

[28] J. M. Goncalves, M. A., M. A. Dahleh, A. Megretski, and M. A. Dahleh, "Global analysis of piecewise linear systems using impact maps and surface Lyapunov functions," Autom. Control. IEEE Trans., vol. 48, no. 12, pp. 2089-2106, dec 2003.

[29] T. E. Chamberlain, H. L. Cole, R. G. Dutton, G. C. Greene, and J. E. Tillman, "Atmopspheric measurements on mars: the viking meteorology experiment," Bulletin of the American Meteorological Society, vol. 57, no. 9, pp. 1094-1104, 1976. 